\title{
Vasopressin and hydration play a major role in the development of glucose intolerance and hepatic steatosis in obese rats
}

\author{
Christopher Taveau • Catherine Chollet • Ludovic Waeckel • Dorinne Desposito • \\ Daniel G. Bichet • Marie-Françoise Arthus • Christophe Magnan • \\ Erwann Philippe • Valerie Paradis • Fabienne Foufelle • Isabelle Hainault • \\ Sofia Enhorning • Gilberto Velho • Ronan Roussel • Lise Bankir • \\ Olle Melander • Nadine Bouby
}

Received: 5 November 2014 / Accepted: 22 December 2014 / Published online: 27 January 2015

(C) Springer-Verlag Berlin Heidelberg 2015

\begin{abstract}
Aims/hypothesis High plasma copeptin, a marker of vasopressin (VP) secretion, has been shown to be associated with the metabolic syndrome and development of type 2 diabetes in humans. The present study was designed to determine the long-term influence of plasma VP concentration in a rodent model prone to metabolic dysfunction.

Methods Obese Zucker rats and their lean counterparts were submitted for 4 weeks to one of three protocols inducing different levels of VP. Circulating VP was either reduced by increasing the daily water intake (low-VP), or increased by a chronic i.p. infusion of VP (high-VP). The control rats had normal VP levels that depended on their own regulation of water intake and VP secretion.
\end{abstract}

Electronic supplementary material The online version of this article (doi:10.1007/s00125-015-3496-9) contains peer-reviewed but unedited supplementary material, which is available to authorised users.

C. Taveau $\cdot$ C. Chollet $\cdot$ L. Waeckel $\cdot$ D. Desposito $\cdot$ F. Foufelle $\cdot$

I. Hainault $\cdot$ G. Velho $\cdot$ R. Roussel $\cdot$ L. Bankir $\cdot$ N. Bouby $(\square)$

Inserm U1138, Centre de Recherche des Cordeliers, 15 rue de l'Ecole

de Medecine, 75006 Paris, France

e-mail: nadine.bouby@crc.jussieu.fr

C. Taveau $\cdot$ C. Chollet $\cdot$ L. Waeckel $\cdot$ D. Desposito $\cdot$ F. Foufelle $\cdot$

I. Hainault $\cdot$ G. Velho $\cdot$ L. Bankir $\cdot$ N. Bouby

Pierre \& Marie Curie University, Paris, France

C. Taveau $\cdot$ C. Chollet $\cdot$ L. Waeckel $\cdot$ D. Desposito $\cdot$ F. Foufelle $\cdot$

I. Hainault $\cdot$ G. Velho $\cdot$ L. Bankir $\cdot$ N. Bouby

Paris Descartes University, Paris, France

D. G. Bichet • M.-F. Arthus

Department of Physiology and Medicine, Sacré-Coeur Hospital,

Montreal, Canada

C. Magnan • E. Philippe

CNRS UMR 8251, Paris, France
Results Compared with controls with normal VP, lean rats with high-VP had a higher fasting glycaemia after 4 weeks. In obese rats, high-VP promoted hyperinsulinaemia, glucose intolerance, assessed by glucose and insulin tolerance tests, and an impaired response to a pyruvate challenge. Conversely, treatment with a selective arginine vasopressin receptor $1 \mathrm{~A}$ (V1aR) antagonist reduced glucose intolerance. Low-VP obese rats had unchanged glucose tolerance but exhibited a drastic decrease in liver steatosis compared with control obese rats, associated with low hepatic triacylglycerol and cholesterol content, and reduced expression of hepatic lipogenic genes. These effects were independent of changes in body adiposity, and plasma sodium and osmolality did not differ among groups.

C. Magnan $\cdot$ E. Philippe $\cdot$ V. Paradis $\cdot$ R. Roussel

Paris Diderot University, Paris, France

V. Paradis

Anatomy and Pathology Department, Beaujon Hospital,

Paris, France

S. Enhorning $\cdot$ O. Melander

Department of Clinical Sciences, Malmö, Lund University,

Malmö, Sweden

S. Enhorning $\cdot$ O. Melander

Department of Internal Medicine, Malmö, Skåne University

Hospital, Malmö, Sweden

R. Roussel

Department of Diabetology-Endocrinology-Nutrition, DHU FIRE, Bichat Hospital, AP-HP, Paris, France 
Conclusion/interpretation These findings show a causal relationship between the VP-hydration axis and the metabolic risk. Therapeutic perspectives include diet recommendations regarding hydration, but also potential pharmacological interventions targeting the VP V1aR.

Keywords Glucose metabolism · Hydration · Lipid metabolism $\cdot$ Liver $\cdot$ Vasopressin

\begin{tabular}{ll}
\multicolumn{2}{l}{ Abbreviations } \\
IR & Insulin resistance \\
ITT & Insulin tolerance test \\
Norm-VP & Normal vasopressin \\
V1aR & Vasopressin V1a receptor \\
V1bR & Vasopressin V1b receptor \\
VP & Vasopressin
\end{tabular}

\section{Introduction}

Although it has been known for several decades that plasma vasopressin (VP) is increased in patients with type 1 or with type 2 diabetes mellitus [1] and in animal models of diabetes $[2,3]$, only recently has the VP-hydration axis emerged as a risk factor for impaired glucose homeostasis and diabetes. An increasing body of data suggests that VP may play a part in glucose homeostasis, besides its well-defined role in urine concentration and body water homeostasis. Acute VP infusion in the rat and in healthy individuals was shown to induce a transient rise in blood glucose concentration [4-6]. Ex vivo studies showed that VP stimulates gluconeogenesis and glycogenolysis through the activation of hepatic arginine VP receptor $1 \mathrm{~A}(\mathrm{~V} 1 \mathrm{aR})$, and the release of either glucagon or insulin, depending on concomitant extracellular glucose levels, through the activation of arginine vasopressin receptor 1B (V1bR) in pancreatic islets [7-9]. Furthermore, VP stimulates adrenocorticotropin hormone release through the activation of pituitary V1bR [10, 11]. The finding that mice with deleted V1aR and/or V1bR exhibit metabolic disorders [12-15] has prompted several epidemiological investigations in humans.

Plasma copeptin, a stable surrogate marker for VP derived from the same preprohormone [16], has been shown to be associated with insulin resistance, metabolic syndrome and obesity [17]. High plasma copeptin predicted future diabetes mellitus independently of known risk factors [18]. The secretion of VP and copeptin strongly depends on the level of hydration. We reported that water intake was inversely and independently associated with the risk of developing hyperglycaemia in a cohort of the general French population [19]. Additionally, VP was shown to contribute to albuminuria and to diabetes-induced nephropathy in animal models [20, 21]. Epidemiological studies in humans are consistent with these findings [21-23].
These observations suggest that a high level of circulating VP has adverse consequences on glucose metabolism, although a causal link between a sustained high level of VP and disorders of glucose homeostasis has not been established. The present investigation was designed to evaluate the longterm impact on glucose homeostasis of various levels of circulating VP. The experiments were performed in obese Zucker rats, a genetic model of insulin resistance, and in their lean controls. VP level was altered chronically in both directions, by either VP infusion or an increase in water intake. We showed that VP impaired tolerance to a glucose load, but also induced a marked rise in plasma glucose after pyruvate administration. Blockade of $\mathrm{V} 1 \mathrm{aR}$ reduced glucose intolerance. Unexpectedly, a reduction in plasma VP led to improvement of hepatic lipid metabolism and liver steatosis.

\section{Methods}

Animals and treatments All animal procedures were conducted in accordance with European guidelines for the care and use of laboratory animals (L 358-86/609/EEC). Male 7-weekold lean $(\mathrm{Fa} / \mathrm{fa})$ and obese $(\mathrm{fa} / \mathrm{fa})$ Zucker rats (Charles River Laboratories, l'Arbresle, France) were used. Rats of each strain were submitted for 4 weeks to one of three protocols inducing different levels of VP. Circulating VP was either reduced (low-VP) by increasing water intake (mixing the food with a water-rich agar gel, giving $3 \mathrm{ml}$ water and $40 \mathrm{mg}$ agar per $g$ food [24]) or increased (high-VP) by i.p. infusion of VP (500 ng kg ${ }^{-1}$ day $^{-1}$, Sigma Aldrich, St Quentin, France) by osmotic Alzet minipumps (Charles River, France). The third group had normal VP levels (norm-VP) that depended on their own regulation of water intake and VP secretion.

In a separate series of experiments, obese rats treated with the selective non-peptide V1aR antagonist SR49059 (Relcovaptan; Sanofi-Aventis, Toulouse, France, $30 \mathrm{mg} \mathrm{kg}^{-1}$ day $^{-1}$ mixed in the food) for 4 weeks, were compared with obese untreated rats (controls).

All rats were housed in individual metabolism cages (Techniplast, Louviers, France) and had free access to demineralised water. To ensure the same food intake in all groups, all rats were offered a daily amount of standard diet (A04, Safe, Augy, France) slightly less than their spontaneous intake ( 20 or $25 \mathrm{~g} /$ day to lean or obese rats, respectively).

Plasma and urinary parameters Blood samples were withdrawn from the tail tip and urine samples were collected every second week. Data from two successive $24 \mathrm{~h}$ urine collections were averaged for each rat. The following parameters were measured: urine and plasma osmolality (freezing point osmometer, Roebling, Berlin, Germany), urinary VP concentration (RIA as described previously [25]), plasma concentration of sodium and potassium (flame photometer, Instrumentation 
Laboratory, Lexington, MA, USA), triacylglycerol and total cholesterol (automatic analyser Konelab201, ThermoElectron, Ilkirch, France) and apelin (ELISA, Phoenix Pharmaceuticals, Strasbourg, France).

Glucose and insulin tolerance and pyruvate tests After 2 and 4 weeks of treatment, glucose tolerance tests (GTTs; $1.5 \mathrm{~g} / \mathrm{kg}$, i.p.) and insulin tolerance tests (ITTs; $1 \mathrm{U} / \mathrm{kg}$, i.p.) were performed after a $6 \mathrm{~h}$ fasting period. Each test was separated by two rest days. Blood samples were collected from the tail tip at the indicated times. Glucose concentrations were measured (Diagnostic Glucometer, Lifescan Laboratories, Issy-lesMoulineaux, France) and insulin and glucagon concentrations were assayed by ELISA (Mercodia, Uppsala, Sweden). A pyruvate test was performed $(1 \mathrm{~g} / \mathrm{kg}$, i.p. $)$ in another series of three groups of obese rats treated for 2 weeks as described above. The insulin resistance (IR) index and AUC were calculated (see electronic supplementary material [ESM] Methods).

Fat/lean body mass measurements Body composition was measured in live animals by nuclear magnetic resonance imaging (EchoMRI 900, Houston, TX, USA).

Analysis of hepatic gene expression Total RNA extraction, cDNA synthesis and real-time PCR were carried out to assess the expression of genes encoding V1aR (Avprla, alias V1aR), glucose-6-phosphatase (G6pc alias G6Pase), glycogen phosphorylase liver form (Pygll), phosphoenolpyruvate carboxykinase (Pck1, alias Pepck), glucose transporters 1 (Scl2al) and 2 (Scl2a2), aquaporin 9 (Aqp9), fatty acid synthase ( $F a s n$, alias Fas), sterol regulatory element binding protein 1 (Srebf1, alias Srebp-1c) and 2 (Srebf2, alias Srebp-2c) (see ESM for further details). Data were normalised to $18 \mathrm{~S}$ rRNA. Changes in the target genes relative to their mean expression in the norm-VP group were calculated by the $2^{-\Delta \Delta \mathrm{C}_{\mathrm{t}}}$ comparative method [26].

Histological analysis Small pieces of liver tissue were frozen at $-80^{\circ} \mathrm{C}$ and routinely processed for histopathological analysis. Sections of tissue, $5 \mu \mathrm{m}$ thick, were stained with haematoxylin-eosin and Sirius Red. Steatosis, necroinflammation as well as portal and perisinusoidal fibrosis were assessed by light microscopy.

Hepatic triacylglycerol, cholesterol and glycogen content Triacylglycerol and cholesterol liver content was determined using commercial kits (Randox Laboratories, Crumlin, UK). Liver glycogen content was determined according to Bergmeyer [27]. Briefly, $100 \mathrm{mg}$ liver was homogenised in $0.2 \mathrm{~mol} / \mathrm{l}$ acetate buffer and incubated in the presence of $\alpha-(1-4),(1-6)$-amyloglucosidase (Roche Diagnostics, Meylan, France) for $1 \mathrm{~h}$ at $55^{\circ} \mathrm{C}$. Glucose release was measured by enzymatic spectrophotometric assays.
Statistical analysis Data are expressed as mean \pm SEM. Statistical analyses were performed by one-way ANOVA with Fisher protected least significant difference post hoc test or paired Student's $t$ test. Values of $p<0.05$ were considered significant. Results obtained in obese and lean strains were analysed independently.

\section{Results}

Effect of chronic VP modulation on body growth and water homeostasis in lean and obese Zucker rats To determine if VP is involved in the regulation of glucose homeostasis under insulin-sensitive and insulin-resistant conditions, we examined the effect of three different levels of circulating VP, first in Zucker lean and then in Zucker obese rats. The physiological characteristics of lean and obese rats after 2 weeks of treatment are presented in Tables 1 and 2, respectively. Plasma and urine data after 4 weeks of treatment were not different from those measured on the second week (not shown).

All rats of each genotype grew regularly during the experiment. There was no difference in body weight among the groups. Treatments did not change the proportion of body fat in obese rats after 4 weeks of treatment $(48 \pm 1,46 \pm 1,44$ $\pm 1 \%$ relative to body weight in low-VP, norm-VP and highVP groups, respectively).

The chronic increase in water intake lowered urinary VP excretion in low-VP rats to about one-tenth that in control norm-VP rats in both obese and lean rats. The infusion of VP resulted in a 10- to 25 -fold increase in daily urinary VP excretion in high-VP rats of both genotypes compared with

Table 1 Physiological parameters of lean Zucker rats after 2 weeks of treatment

\begin{tabular}{|c|c|c|c|}
\hline Characteristic & Low-VP & Norm-VP & High-VP \\
\hline Body weight (g) & $280 \pm 9$ & $284 \pm 10$ & $270 \pm 6$ \\
\hline Water intake (ml/day) & $62.2 \pm 0.5^{* * *, \dagger \dagger}$ & $17.8 \pm 0.7$ & $18.4 \pm 0.5$ \\
\hline $\begin{array}{l}\text { Urine VP excretion } \\
\text { (ng/day) }\end{array}$ & $1.4 \pm 0.2^{* * *, \dagger \dagger}$ & $11.0 \pm 1.5$ & $264 \pm 39 * * *$ \\
\hline Urine volume ( $\mathrm{ml} /$ day) & $29.5 \pm 0.7 * * *, \dagger \dagger$ & $6.3 \pm 0.5$ & $6.5 \pm 0.2$ \\
\hline $\begin{array}{l}\text { Urine osmolality } \\
\left(\text { mosm } / \mathrm{kg} \mathrm{H}_{2} \mathrm{O}\right)\end{array}$ & $473 \pm 15^{* * *, \dagger \dagger}$ & $2,320 \pm 78$ & $2,296 \pm 78$ \\
\hline Haematocrit (\%) & $50.2 \pm 0.7$ & $48.0 \pm 1.2$ & $49.3 \pm 0.7$ \\
\hline $\begin{array}{l}\text { Plasma osmolality } \\
\left(\operatorname{mosm} / \mathrm{kg} \mathrm{H}_{2} \mathrm{O}\right)\end{array}$ & $297 \pm 1$ & $297 \pm 1$ & $296 \pm 1$ \\
\hline Plasma $\mathrm{Na}^{+}(\mathrm{mmol} / \mathrm{l})$ & $147 \pm 1$ & $146 \pm 1$ & $147 \pm 1$ \\
\hline Plasma K ${ }^{+}(\mathrm{mmol} / \mathrm{l})$ & $4.7 \pm 0.3$ & $4.9 \pm 0.2$ & $5.0 \pm 0.3$ \\
\hline
\end{tabular}

Results are mean \pm SEM; $n=7-8$ /group

ANOVA followed Fisher's post hoc test: low-VP or high-VP vs norm-VP, ${ }^{* * *} p<0.001$; low-VP vs high-VP, ${ }^{\dagger \dagger} p<0.001$ 
Table 2 Physiological parameters of obese Zucker rats after 2 weeks of treatment

\begin{tabular}{|c|c|c|c|}
\hline Characteristic & Low-VP & Norm-VP & High-VP \\
\hline Body weight (g) & $315 \pm 8$ & $317 \pm 8$ & $309 \pm 6$ \\
\hline Water intake (ml/day) & $88.0 \pm 4.0 * * *, \dagger \dagger$ & $17.4 \pm 1.1$ & $18.0 \pm 1.1$ \\
\hline $\begin{array}{l}\text { Urine VP excretion } \\
\text { (ng/day) }\end{array}$ & $0.9 \pm 0.3^{* * *, \dagger \dagger \dagger}$ & $14.3 \pm 1.8$ & $96.6 \pm 13.5^{* * *}$ \\
\hline Urine volume (ml/day) & $49.9 \pm 4.2 * * *, \dagger \dagger$ & $10.8 \pm 0.7$ & $10.5 \pm 0.5$ \\
\hline $\begin{array}{l}\text { Urine osmolality } \\
\qquad\left(\mathrm{mosm} / \mathrm{kg} \mathrm{H}_{2} \mathrm{O}\right)\end{array}$ & $582 \pm 32 * * *, \dagger \dagger$ & $2,484 \pm 71$ & $2,538 \pm 61$ \\
\hline Haematocrit (\%) & $44.4 \pm 1.6$ & $43.4 \pm 1.8$ & $43.1 \pm 1.2$ \\
\hline $\begin{array}{l}\text { Plasma osmolality } \\
\left.\text { (mosm } / \mathrm{kg} \mathrm{H}_{2} \mathrm{O}\right)\end{array}$ & $303 \pm 3$ & $303 \pm 5$ & $301 \pm 4$ \\
\hline Plasma $\mathrm{Na}^{+}(\mathrm{mmol} / \mathrm{l})$ & $144 \pm 1$ & $141 \pm 1$ & $142 \pm 1$ \\
\hline Plasma K ${ }^{+}(\mathrm{mmol} / \mathrm{l})$ & $5.2 \pm 0.4$ & $5.6 \pm 0.6$ & $5.5 \pm 0.6$ \\
\hline $\begin{array}{l}\text { Plasma triacylglycerol } \\
(\mathrm{mmol} / \mathrm{l})\end{array}$ & $0.59 \pm 0.04$ & $0.63 \pm 0.06$ & $0.48 \pm 0.07$ \\
\hline $\begin{array}{l}\text { Plasma cholesterol } \\
\text { (mmol/l) }\end{array}$ & $3.45 \pm 0.15$ & $4.37 \pm 0.26$ & $3.34 \pm 0.41$ \\
\hline Plasma apelin (ng/ml) & $4.9 \pm 0.3$ & $4.4 \pm 0.3$ & $3.8 \pm 0.3$ \\
\hline
\end{tabular}

Results are mean \pm SEM; $n=7-8$ /group

ANOVA followed by Fisher's post hoc test: high-VP or low-VP vs normVP, $* * * p<0.001$; low-VP vs high-VP, ${ }^{\dagger \dagger} p<0.001$

norm-VP rats. These values are comparable with those observed after $24 \mathrm{~h}$ of dehydration in normal rats [28].

As expected, water intake and urine volume were markedly higher in low-VP than in the norm-VP group. In contrast, VP infusion did not change fluid intake, urine volume and osmolality in the high-VP group compared with the norm-VP group. This is probably due to the attenuation of anti-diuretic/anti-natriuretic arginine $\mathrm{VP}$ receptor 2 (V2R)-mediated effects by activation of the tubular V1aR [28]. Daily osmolar excretion was comparable in all three groups, as expected from the equivalent food intake imposed on all rats. There was no significant difference in plasma osmolality, natraemia, kaliaemia or haematocrit between the different groups.

High VP level increases fasting glycaemia and impairs glucose tolerance in lean rats Chronic high VP level resulted in a progressive rise in fasting glycaemia in lean rats (Fig. 1a). At week 4, the glycaemic difference between high- and norm-VP groups amounted to $1.5 \mathrm{mmol} / \mathrm{l}$ paralleled by fourfold higher values of insulinaemia in the high-VP group ( $146 \pm 26$ vs $34 \pm$ $9 \mathrm{pmol} / \mathrm{l}, p<0.05)$. Consequently, the IR index was fourfold higher in the high-VP than in the norm-VP groups of rats. Glucose tolerance was significantly impaired in the high-VP group as demonstrated by the GTT results (Fig. 1b). Low-VP level slightly decreased fasting glycaemia but it did not change the glucose response during GTT compared with the norm-VP group (Fig. 1a, b).
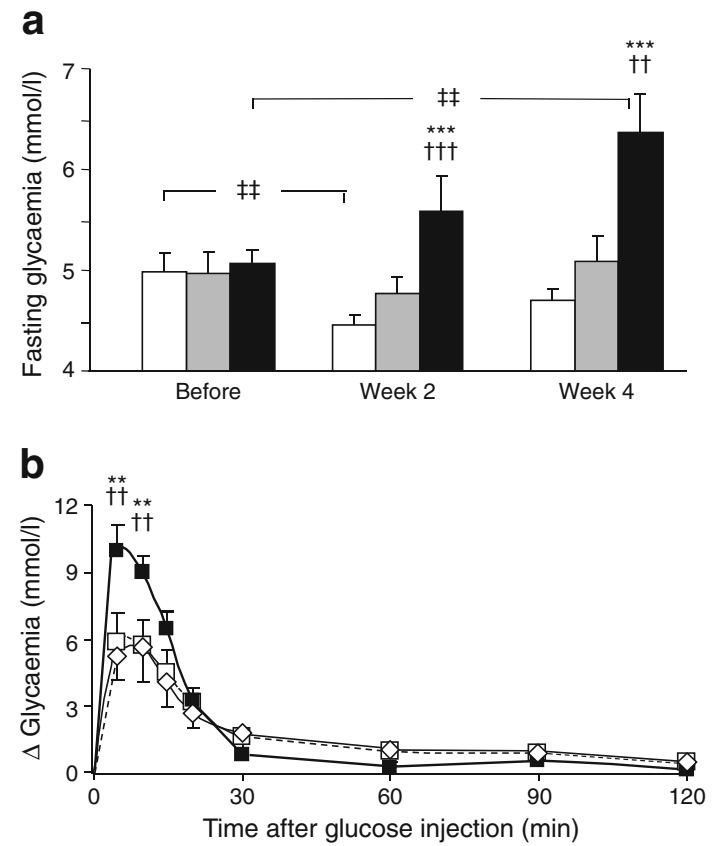

Fig. 1 High VP level increases glycaemia and alters glucose tolerance in lean Zucker rats. (a) Time-dependent effect of VP on fasting glycaemia in rats with low (white bars), normal (grey bars) and high (black bars) VP level. (b) Glycaemic response during i.p. GTT performed 4 weeks after onset of treatments inducing low (white diamonds, continuous line), normal (white squares, dashed line) and high VP level (black squares, thick line). Data are expressed as changes relative to time 0 . Values are expressed as mean \pm SEM; $n=6 /$ group. ANOVA followed by Fisher's post hoc test: high-VP vs norm-VP, ${ }^{* *} p<0.01$ and $* * * p<0.001$; highVP vs low-VP, ${ }^{\dagger} p<0.01$ and ${ }^{\dagger \dagger} p<0.001$; time effect, ${ }^{+*} p<0.01$

High VP level aggravates disorders of glucose metabolism in obese insulin-resistant rats No differences were observed in the plasma concentrations of triacylglycerol or total cholesterol or in fasting blood glycaemia (Table 2, Fig. 2a) between the differently treated groups of obese rats. However, chronic VP infusion induced a significant increase in fasting insulinaemia $(674 \pm 107,1,449 \pm 112,1,217 \pm 65 \mathrm{pmol} / 1$ before, and after 2 or 4 weeks of treatment, respectively, $p<0.001$ ) (Fig. 2b). Thus, the IR index was already $50 \%$ higher in the high-VP group than in the two other groups after 2 weeks of treatment (Fig. 2c). At this time point, an i.p. glucose load induced a twofold higher level of glycaemia and insulinaemia response in the high-VP group compared with the other groups (Fig. 2d, e and Table 3). Similar results were obtained after 4 weeks of treatment (Table 3 ).

To understand the effect of VP on glucose tolerance, we assessed the effect of VP on hepatic gluconeogenic capacity with a pyruvate test. The glucose excursion in the next $2 \mathrm{~h}$ was markedly increased by high-VP treatment, peaking with a twofold greater glycaemia than in the other groups (Fig. 2f). Treatments did not change the mRNA abundance of genes coding for enzymes involved in gluconeogenesis and glycogenolysis or the expression of hepatic glucose transporters (see ESM Table 1). Obese high-VP rats exhibited a 50\% 
Fig. 2 High VP level aggravates glucose intolerance in obese Zucker rats. (a-c) Fasting glycaemia and insulinaemia and calculated IR index in rats with low (white bars), normal (grey bars) and high (black bars) VP level. (d, e) Intraperitoneal GTT and (f) pyruvate challenge were performed 2 weeks after onset of treatment inducing low (white diamonds, continuous line), normal (white squares, dashed line) or high VP level (black squares, thick line). Data are expressed as changes relative to time 0 . Values are expressed as mean \pm SEM; $n=8$ /group. ANOVA followed by Fisher's post hoc test: high-VP vs norm$\mathrm{VP}, * * p<0.01$ and $* * * p<0.001$; high-VP vs low-VP, ${ }^{\dagger \dagger} p<0.01$ and ${ }^{\dagger \dagger} p<0.001$; time effect, ${ }^{*} p<0.01$ and ${ }^{* t} p<0.001$
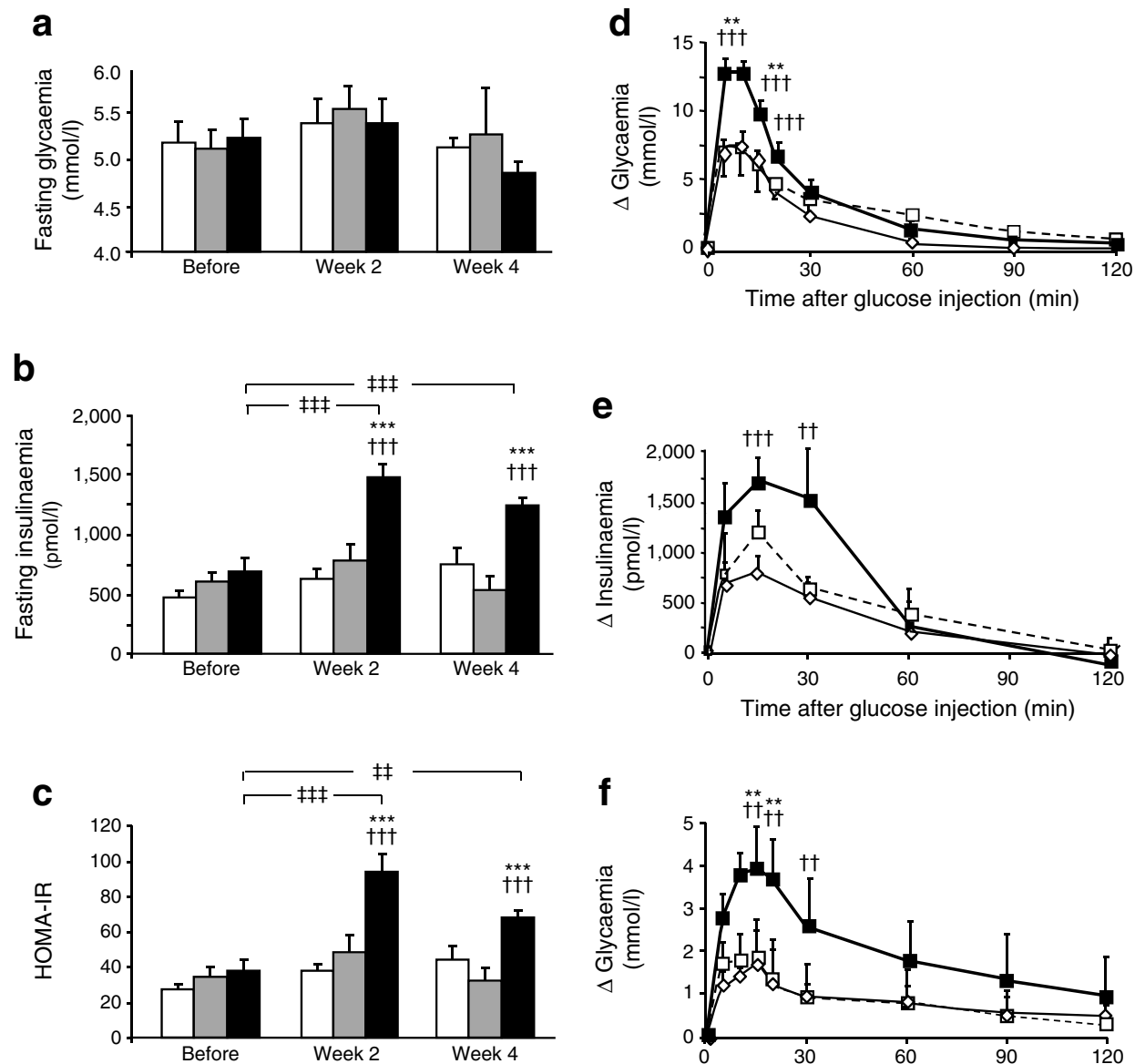

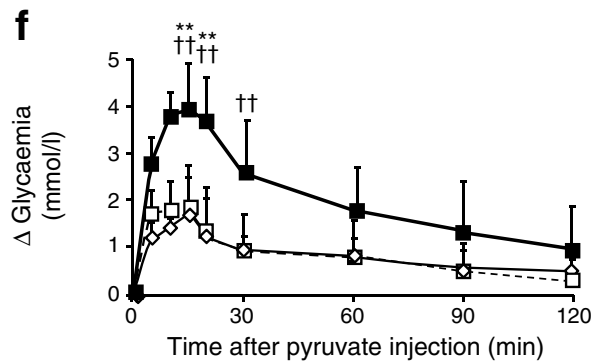

Table 3 AUCs of responses to glucose, insulin or pyruvate test in obese Zucker rats with low, normal or high VP level

\begin{tabular}{llll}
\hline Test & Low-VP & Norm-VP & High-VP \\
\hline GTT week 2 & & & \\
$\quad$ Glycaemia (mmol/1.2 h) & $153 \pm 25$ & $174 \pm 38$ & $335 \pm 23^{* * *, \dagger \dagger \dagger}$ \\
$\quad$ Insulinaemia (nmol/1.2 h) & $34 \pm 4$ & $40 \pm 6$ & $79 \pm 18^{* *, \dagger \dagger}$ \\
GTT week 4 & & & \\
$\quad$ Glycaemia (mmol/1.2 h) & $300 \pm 46$ & $391 \pm 44$ & $524 \pm 38^{*}, \dagger \dagger$ \\
$\quad$ Insulinaemia (nmol/1.2 h) & $72 \pm 6$ & $56 \pm 14$ & $127 \pm 21^{* *, \dagger}$ \\
ITT week 2 & & & \\
$\quad$ Glycaemia (mmol/1.2 h) & $316 \pm 15^{* * *}$ & $146 \pm 15$ & $172 \pm 19^{\dagger \dagger \dagger}$ \\
$\quad$ Insulinaemia (nmol/1.2 h) & $168 \pm 27^{*}$ & $254 \pm 36$ & $314 \pm 12^{* *}$ \\
$\quad$ Glucagonaemia (pmol/1.2 h) & $1,274 \pm 57$ & $1,304 \pm 39$ & $1,260 \pm 61$ \\
ITT week 4 & & & \\
$\quad$ Glycaemia (mmol/1.2 h) & $134 \pm 25$ & $113 \pm 24$ & $130 \pm 18$ \\
Pyruvate test week 2 & & & \\
$\quad$ Glycaemia (mmol/1.2 h) & $832 \pm 97$ & $805 \pm 34$ & $989 \pm 98$ \\
\hline
\end{tabular}

Results are mean \pm SEM; $n=5$-8/group

ANOVA followed by Fisher's post hoc test: high-VP or low-VP vs norm$\mathrm{VP},{ }^{*} p<0.05,{ }^{* *} p<0.01$ and ${ }^{* * *} p<0.001$; high-VP vs low-VP, ${ }^{\dagger} p<0.05$, ${ }^{\dagger} p<0.01$ and ${ }^{\dagger \dagger} p<0.001$ higher Avprla mRNA abundance than the norm-VP rats $(1.52 \pm 0.15$ vs $1.00 \pm 0.15, p<0.01)$, while low-VP rats tended to show a $25 \%$ decline $(0.73 \pm 0.13$, non-significant $)$.

During ITT, plasma insulin levels reached significantly higher values in high-VP rats than in other rats 15 min after insulin injection $(4,138 \pm 130,3,194 \pm 4,047$ and $2,419 \pm$ $426 \mathrm{pmol} / \mathrm{l}$ in high-VP, norm-VP, and low-VP groups, respectively, $p<0.03$ ) because of differences in basal values (Fig. 3b). Despite these differences in insulinaemia, the initial fall in glycaemia was similar between groups (Fig. 3a), suggesting a lower sensitivity to insulin in high-VP animals that was partly evidenced by calculation of the IR index. Glycaemia returned faster to baseline level in high-VP rats than in norm-VP rats, resulting in slightly lower AUC values (Table 3). Sustained hypoglycaemia persisted in low-VP rats throughout the test, with significantly lower values of glycaemia at time points 90 and $120 \mathrm{~min}(p<0.01)$ (Fig. 3a). These different patterns in the late portion of the ITT suggest differences in the counter-regulatory responses to insulin-induced hypoglycaemia. However, the main candidate for such counter-regulation, i.e. glucagon, showed similar changes in concentration following hypoglycaemia in all groups (Fig. 3c). 
a

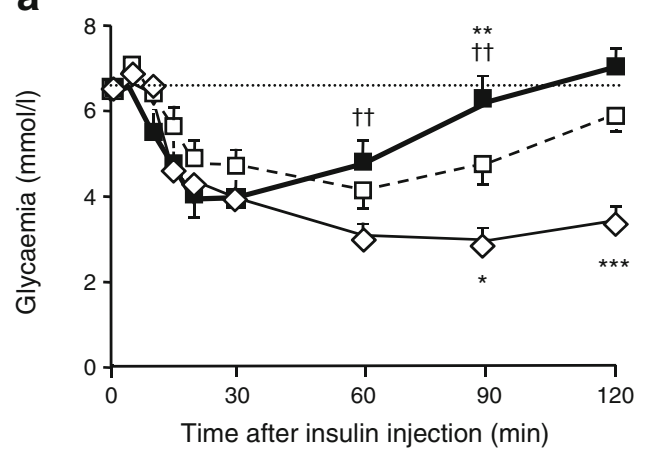

b
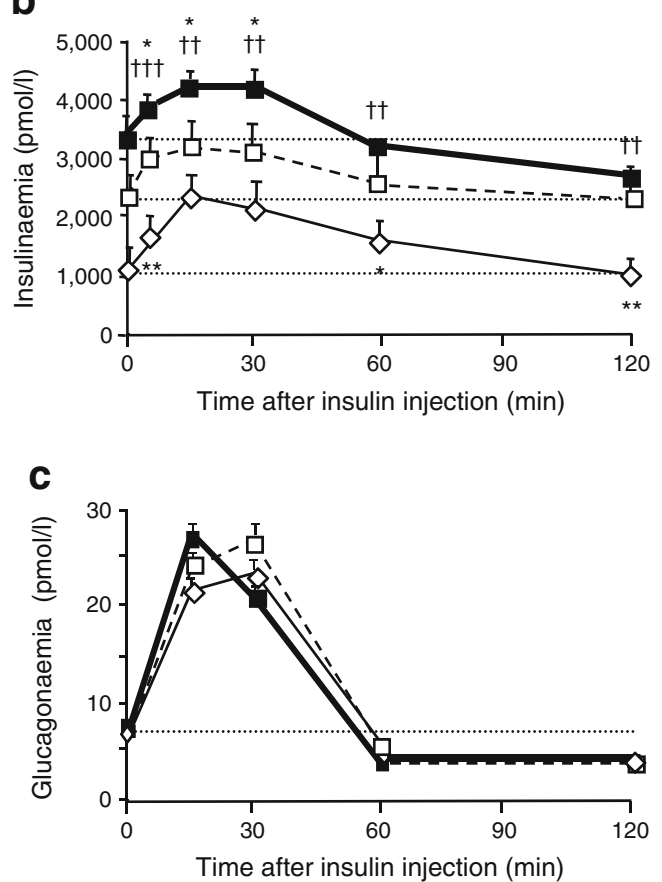

Fig. 3 Effect of VP level on (a) glycaemic, (b) insulinaemic and (c) glucagonaemic responses during ITT in obese Zucker rats. Intraperitoneal ITT was performed 2 weeks after onset of treatment inducing low (white diamonds, continuous line), normal (white squares, dashed line) or high VP level (black squares, thick line). Dotted lines indicate the initial basal levels. Values are expressed as mean $\pm \mathrm{SEM} ; n=7 /$ group. ANOVA followed by Fisher's post hoc test: high-VP or low-VP vs norm-VP, ${ }^{*} p<0.05$ and $* * p<0.01$; high-VP vs low-VP, ${ }^{\dagger \dagger} p<0.01$ and ${ }^{\dagger \dagger} p<0.001$

VlaR blockade partially restores glucose tolerance in obese rats To determine if V1aR are involved in the disorders of glucose metabolism in obese Zucker rats, we examined the effect of chronic V1aR antagonism. No difference in body growth or parameters of water metabolism was observed between the rats receiving V1aR-antagonist and control rats (see ESM Table 2). After 4 weeks of treatment, fasting blood glycaemia levels were not significantly different between the groups $(5.34 \pm 0.21$ vs $5.16 \pm 0.16 \mathrm{mmol} / 1$ in the V1aRantagonist and control groups, respectively) but fasting insulinaemia was significantly lower in the V1aR-antagonist group than in the control group $(643 \pm 180$ vs $1,526 \pm$ $163 \mathrm{pmol} / 1, p<0.001)$. Consequently, the IR index was reduced to about half in the V1aR-antagonist group compared with the non-treated control group ( $23 \pm 7$ vs $49 \pm 4, p<0.01$ ). During the GTT, the amplitude of glycaemic response was significantly decreased by $35 \%$ in the V1aR-antagonist group compared with the control group, whereas the amplitude of insulin response was not significantly different between the groups (Fig. 4a, b). Insulin i.p. injection induced a similar pattern of glycaemic changes in both groups (Fig. 4c).

Low-VP protects liver tissue from steatosis in obese rats by preventing upregulation of lipogenesis Histological analysis of liver showed that high-VP and norm-VP obese rats had similar degrees and types of steatosis, characterised by mediovesicles and macrovacuoles mainly localised in the centro- and medio-lobular areas. Mild necroinflammation was observed. Neither portal nor perisinusoidal fibrosis was detected in the different groups. Interestingly, low-VP rats with high hydration had almost no steatosis after 2 weeks of treatment (Fig. 5a).
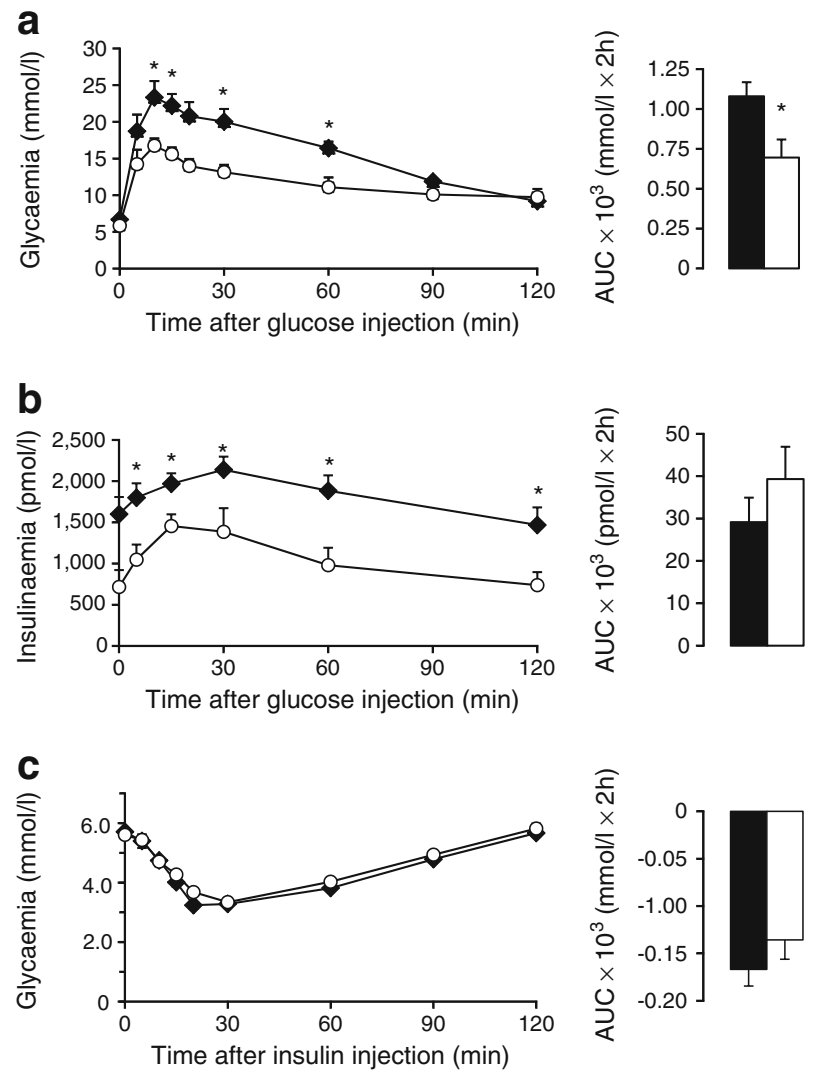

Fig. 4 V1aR antagonism attenuates glucose intolerance in obese Zucker rats. (a, b) Glycaemic and insulinaemic responses during i.p. GTT and (c) glycaemic responses during i.p. ITT in V1aR antagonist-treated rats (white circles, white bars) and in control untreated rats (black diamonds, black bars). Bar graphs are the AUCs of GTT or ITT calculated from the original kinetic graphs. Values are expressed as mean $\pm \mathrm{SEM} ; n=8$ /group. Student's $t$ test: ${ }^{*} p<0.05$ 
Hepatic lipid content paralleled the histological changes. Low-VP/high hydration markedly decreased triacylglycerol and cholesterol content compared with norm-VP-treated animals (Fig. 5b, c) as well as gene expression of proteins involved in lipogenesis, fatty acid synthase (FAS), sterol regulatory element binding protein 1c (SREBP-1c) and aquaporin 9 (AQP-9) (Fig. 5e-h). Low-VP was also associated with an increase in hepatic glycogen content (Fig. 5d).

a
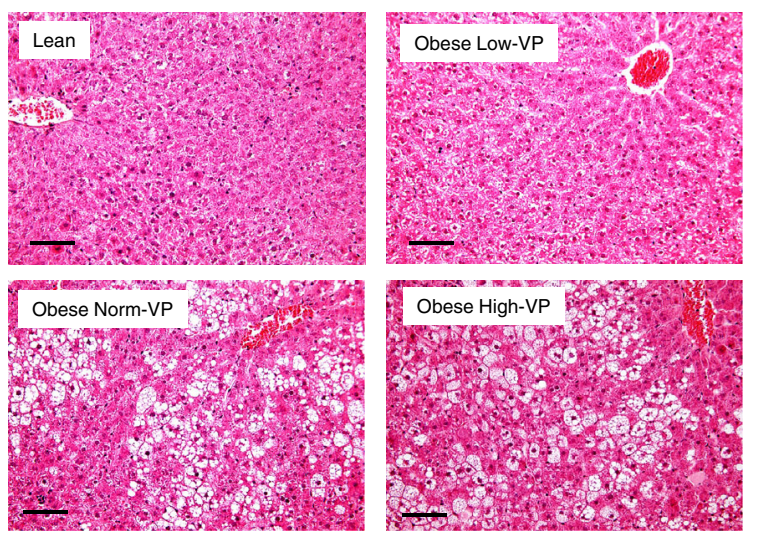

b

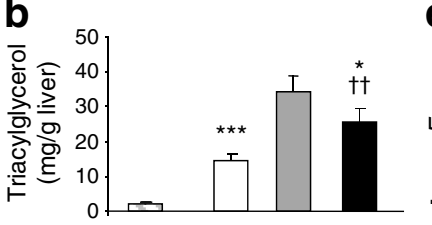

e



C
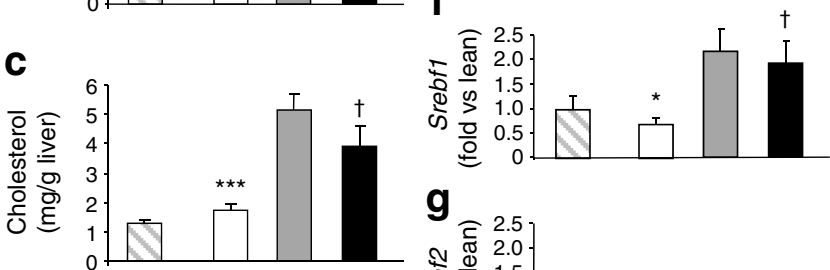

g
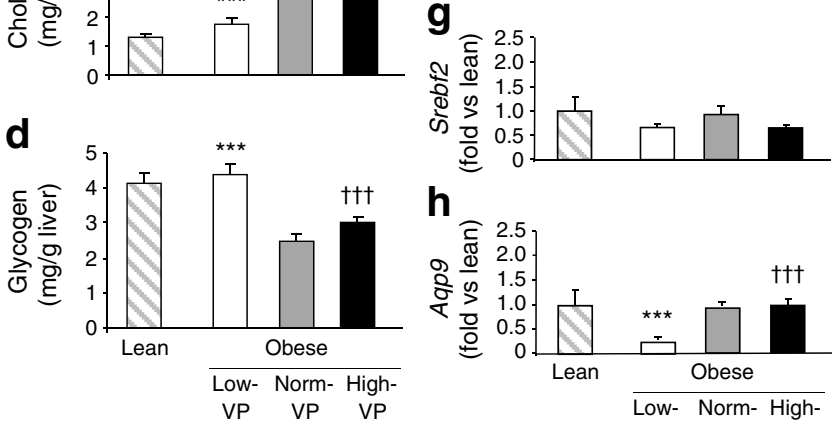

h

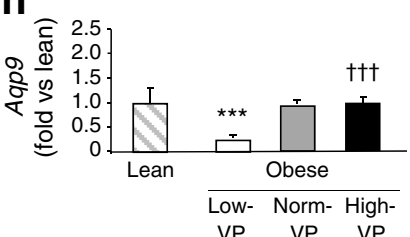

Fig. 5 Low VP level induced by high hydration reverses hepatosteatosis in obese Zucker rats. (a) Representative image of liver tissue. Sections were stained with haematoxylin-eosin and Sirius Red. Scale bar, $100 \mu \mathrm{m}$. (b-d) Hepatic concentration of triacylglycerol, cholesterol and glycogen. (e, f) mRNA levels of proteins involved in fatty acids, cholesterol and triacylglycerol synthesis. Data were obtained 2 weeks after onset of treatment. Values are expressed as mean $\pm \mathrm{SEM} ; n=6$ /group. ANOVA followed by Fisher's post hoc test: high-VP or low-VP vs norm-VP, ${ }^{*} p<0.05$ and $* * * p<0.001$; high-VP vs low-VP, ${ }^{\dagger} p<0.05,{ }^{\dagger \dagger} p<0.01$ and $+\dagger p<0.001$

\section{Discussion}

This work was designed to investigate the chronic effect of VP on glucose homeostasis in rats. Although for several decades the liver was known to express VP receptors, leading Morel et al [29] to choose the rat liver for cloning the first VP receptor by expression cloning more than 20 years ago, no previous study to our knowledge investigated the chronic influence of VP on liver metabolism in vivo. The present study reveals that a sustained high VP level alters glucose metabolism, increasing glycaemia in lean healthy rats, and impairing glucose tolerance in obese rats, at least partly through V1aR. The results of this study point also to a beneficial effect of low VP level and high hydration to alleviate the hepatic steatosis associated with obesity in this model.

Previous studies have evaluated the role of VP and VP receptors on glucose tolerance using VP-deficient rat or mice with inactivation of V1aR and/or V1bR. Conflicting results with arginine vasopressin (AVP)-deficient Brattleboro rats have been reported. Homozygous Brattleboro rats with diabetes insipidus (di/di) showed a decreased plasma glucose level and insulin level when compared with control Long-Evans rats [14] but presented an enhanced glucose tolerance when compared with heterozygous $\mathrm{di} /+$ rats [15]. VlaR-knockout and V1bR (alias Avpr1b)-knockout mice exhibited the opposite phenotype concerning insulin sensitivity $[12,13]$, but the contribution of VP to glucose metabolism seems greater through V1aR than through V1bR. Indeed, mice lacking both receptors and fed a high-fat diet displayed an impaired glucose tolerance similar to that seen in mice with only V1aR inactivation [14]. Noteworthy, these mice presented alterations in plasma volume, body weight gain and energy intake [12, 14]. These features, in addition to the complex interactions between receptor signalling and compensatory mechanisms that occur in the life-long absence of one component of the VP system, could explain the apparent discrepancy with our results.

To study the relationship between VP and glucose metabolism in vivo, we used a different approach. We modified the normal circulating VP concentration in adult rats by either a chronic infusion of VP or an increase in water intake [24], daily food composition and consumption remaining equal between groups. The high water intake increased the daily urine volume markedly, but not enough to induce the formation of hypo-osmotic urine. Rats excreted this extra water appropriately as no sign of plasma dilution appeared, as indicated by unchanged plasma sodium concentration and haematocrit. This 'bidirectional' protocol mimicked the wide interindividual variability of the usual VP concentration and osmotic thresholds for thirst and VP secretion observed in humans [30]. To our knowledge, this is the first study assessing the role of chronic alterations in VP level on glucose metabolism. 
Our results show that exposure to a high VP level induces disorders in glucose metabolism as early as 2 weeks after onset of the VP treatment in both lean and obese Zucker rats. High VP induced a moderate but significant hyperglycaemic effect in lean rats. In fasted obese rats, high VP induced a marked increase in insulinaemia compared with the control group, with no difference in plasma glucose. Consistent with this observation, GTT and ITT revealed that high VP promotes glucose intolerance and accelerates recovery after insulin-induced hypoglycaemia.

Several mechanisms could account for the impaired glucose tolerance and insulin sensitivity observed during high VP conditions. One is impaired intracellular glucose metabolism due to increased gluconeogenesis and/or increased glycogenolysis as suggested by acute experiments [31, 32], an effect probably related to V1aR activation [29]. This hypothesis is supported by higher glycaemia and increased production of glucose observed during the pyruvate challenge and the upregulation of V1aR in the liver of high-VP rats. Moreover, chronic V1aR antagonist treatment decreased fasting insulinaemia and GTT response in obese rats. This experimental observation is in line with the association reported between a common genetic variant in the human AVPRIA gene and impaired glucose homeostasis in a large population-based sample [33].

Other VP-induced effects on glycogen metabolism could contribute to disorders of glucose metabolism. Vasoconstrictive effects of VP and the resulting decrease in hepatic blood flow could cause hypoxia-driven glycogenolysis [4]. Hepatocytes shrinkage induced by hyperosmolality has been shown to inhibit protein and glycogen synthesis [34]. Thus, although plasma osmolality was not significantly different between the groups, we cannot exclude that a mild alteration in ambient osmolality of insulin target tissues could be an additional factor contributing to glucose intolerance.

Besides insulin, glucagon is a major hormone in glucose homeostasis and plays a role in the pathogenesis of hyperglycaemia in diabetes (as reviewed by Cryer PE [35]). Glucagon secretion could be increased by VP through activation of V1bR in pancreatic islets [7-9], or indirectly by stimulating adrenaline (epinephrine) secretion by chromaffin cells of the adrenal medulla [36]. Crosstalk between V1aR and glucagon receptor and their signalling pathways has been described also in hepatocytes $[37,38]$. However, glucagon is not likely to play a role in the disturbances of glucose metabolism observed in the present study because basal plasma glucagon concentration and glucagon response during ITT were unaltered by chronic VP treatment. In addition to glucagon, the peripheral sympatho-adrenal system plays a key role in the counter-regulatory response to hypoglycaemia. The faster recovery after insulin-induced hypoglycaemia observed in the high-VP group could be related to an alteration in catecholamine secretion, though we did not assess this point in the current study [36, 39]. Moreover, it could be related to a higher metabolic clearance of insulin as suggested by the sharper decline in insulinaemia. Indeed, it has been reported that renal insulin degradation is predominant compared with liver at high insulin concentrations [40] and that VP increases glomerular filtration rate [41, 42].

Steatosis has often been associated with hepatic IR in the context of obesity [43]. Of note, the effects of VP on hepatic lipid accumulation and glucose tolerance are dissociated in our model. Low-VP rats did not display improved glucose tolerance compared with control obese rats but exhibited a marked decrease in hepatic steatosis with low hepatic content in triacylglycerol and cholesterol. High-VP rats displayed impairment of glucose tolerance but no aggravation of hepatic steatosis. A similar dissociation was already reported in some murine models of obesity and non-insulin dependent diabetes mellitus [44, 45].

Previous studies on VP and lipogenesis have reported contradictory results [4, 46-48]. Assimacopoulos-Jeannet et al [48] showed that VP increased lipogenesis in isolated hepatocytes, but when concentration of lactate in the medium was increased, basal lipogenesis was increased and the stimulatory effect of VP was lost. We can speculate that lipogenesis is already maximally stimulated in obese Zucker rats, thus preventing a possible VP-induced additional effect. In contrast, a reduction in circulating VP level may decrease steatosis. The lower mRNA abundance of Fasn and Srebf1 as in the low-VP group suggests a decrease in de novo synthesis of fatty acids, although a decrease in fluxes of nonesterified fatty acids from the adipose tissue to the liver, related to a reduced vasoconstrictive effect of VP, cannot be excluded. This decrease in fatty acid availability, in addition to decreased expression of Aqp 9 , the major pathway of glycerol entry in hepatocytes, is probably responsible, at least in part, for the fall in triacylglycerol content. The unaltered mRNA expression of $\operatorname{Srebf2}$, the key regulator for the cholesterol biosynthesis, suggests that the decrease in cholesterol content in the low-VP group is due to an increase in cholesterol degradation rather than a decrease in synthesis.

Although the effect of high water intake on the reduction in steatosis is likely due to the reduction in VP plasma level, other hormonal changes related to increased water intake could be involved. Apelin, an adipokine with anti-obesity properties, is also a neurohypophyseal hormone, as is VP. Apelin and VP are secreted in response to opposite changes in plasma osmolality and exert opposite effects in acute regulation of water balance both centrally and at the level of the kidney [49-51]. Apelin has been shown to be involved in glucose metabolism disorders and in hepatic fibrosis $[52,53]$. However, the finding of similar concentrations of circulating apelin in the different groups of the present study does not support an apelin-mediated effect. The mechanisms responsible for 
the low-VP/high hydration level effect on liver steatosis remain to be clarified.

In conclusion, our study is the first addressing the chronic metabolic actions of VP in vivo. The VP level was altered in two opposite directions for several weeks in two different rat models (lean and obese). The results of these experimental interventions revealed a significant influence of the VP/ hydration system not only on basal glycaemia and glucose tolerance but also on liver steatosis. They clearly support a causality link in the associations observed in epidemiological studies between high copeptin level or low water intake and the prevalence and incidence of hyperglycaemia, metabolic syndrome and type 2 diabetes. Further studies are required to elucidate the mechanisms responsible for these effects. Overall, these findings suggest that, in people who are at risk of diabetes and have an inappropriate hydration status, a moderate increase in water intake, a costless lifestyle change not exclusive of other recommendations, may help prevent or delay the onset of hyperglycaemia and subsequent diabetes, together with its renal complications. Therapeutic perspectives may include also pharmacological interventions targeted at V1aR.

Acknowledgements We are grateful for the technical assistance of the staff of the phenotyping platform (Cordeliers Research Centre, Paris, France) and more particularly to G. Zadigue.

Parts of this study were presented in abstract form at the congresses of the French Society of Diabetology (Paris, March 2014) and the French Society of Neuroendocrinology (September 2013).

Funding This study was supported by grants from the European Research Council (project 282255 Cardioprevent), the Swedish Heart and Lung Foundation, the Swedish Research Council, the Novo Nordisk Foundation, Inserm, Pierre \& Marie Curie and Paris Descartes Universities.

Duality of interest The authors declare that there is no duality of interest associated with this manuscript.

Contribution statement CT, CC, LW, DD, DGB, M-FA, EP, VP, IH, $\mathrm{SE}$ and GV contributed to the acquisition and analysis of data and helped to review the article. $\mathrm{CM}, \mathrm{FF}, \mathrm{RR}, \mathrm{LB}, \mathrm{OM}$ and $\mathrm{NB}$ contributed to the design and conception of the study, provided data interpretation and wrote the article. All authors approved the final version of the manuscript. NB is the guarantor of this work.

\section{References}

1. Zerbe RL, Vinicor F, Robertson GL (1979) Plasma vasopressin in uncontrolled diabetes mellitus. Diabetes 28:503-508

2. Brooks DP, Nutting DF, Crofton JT, Share L (1989) Vasopressin in rats with genetic and streptozocin-induced diabetes. Diabetes 38:54 57

3. Yi SS, Hwang IK, Kim YN et al (2008) Enhanced expressions of arginine vasopressin (Avp) in the hypothalamic paraventricular and supraoptic nuclei of type 2 diabetic rats. Neurochem Res 33:833-841
4. Hems DA, Whitton PD, Ma GY (1975) Metabolic actions of vasopressin, glucagon and adrenalin in the intact rat. Biochim Biophys Acta 411:155-164

5. Rofe AM, Williamson DH (1983) Metabolic effects of vasopressin infusion in the starved rat. Reversal of ketonaemia. Biochem J 212: 231-239

6. Spruce BA, McCulloch AJ, Burd J et al (1985) The effect of vasopressin infusion on glucose metabolism in man. Clin Endocrinol (Oxf) 22:463-468

7. Lee B, Yang C, Chen TH, al-Azawi N, Hsu WH (1995) Effect of AVP and oxytocin on insulin release: involvement of $\mathrm{V} 1 \mathrm{~b}$ receptors. Am J Physiol 269:E1095-E1100

8. Yibchok-Anun S, Cheng H, Chen TH, Hsu WH (2000) Mechanisms of AVP-induced glucagon release in clonal alpha-cells in-R1-G9: involvement of $\mathrm{Ca}(2+)$-dependent and -independent pathways. Br J Pharmacol 129:257-264

9. Abu-Basha EA, Yibchok-Anun S, Hsu WH (2002) Glucose dependency of arginine vasopressin-induced insulin and glucagon release from the perfused rat pancreas. Metabolism 51:1184-1190

10. Gillies GE, Linton EA, Lowry PJ (1982) Corticotropin releasing activity of the new CRF is potentiated several times by vasopressin. Nature 299:355-357

11. Serradeil-Le Gal C, Wagnon J, Simiand J et al (2002) Characterization of (2S,4R)-1-[5-chloro-1-[(2,4-dimethoxyphenyl)sulfonyl]-3-(2methoxy-phenyl)-2-oxo-2,3-dihydro-1H-indol-3-yl]-4-hydroxy-N, $\mathrm{N}$-dimethyl-2-pyrrolidine carboxamide (SSR149415), a selective and orally active vasopressin V1b receptor antagonist. J Pharmacol Exp Ther 300:1122-1130

12. Aoyagi T, Birumachi J, Hiroyama M et al (2007) Alteration of glucose homeostasis in V1a vasopressin receptor-deficient mice. Endocrinology 148:2075-2084

13. Fujiwara Y, Hiroyama M, Sanbe A et al (2007) Insulin hypersensitivity in mice lacking the V1b vasopressin receptor. J Physiol 584: 235-244

14. Nakamura K, Aoyagi T, Hiroyama M et al (2009) Both V(1A) and $\mathrm{V}(1 \mathrm{~B})$ vasopressin receptors deficiency result in impaired glucose tolerance. Eur J Pharmacol 613:182-188

15. Nakamura K, Yamashita T, Fujiki H et al (2011) Enhanced glucose tolerance in the Brattleboro rat. Biochem Biophys Res Commun 405: 64-67

16. Morgenthaler NG, Struck J, Alonso C, Bergmann A (2006) Assay for the measurement of copeptin, a stable peptide derived from the precursor of vasopressin. Clin Chem 52:112-119

17. Saleem U, Khaleghi M, Morgenthaler NG et al (2009) Plasma carboxy-terminal provasopressin (copeptin): a novel marker of insulin resistance and metabolic syndrome. J Clin Endocrinol Metab 94: $2558-2564$

18. Enhorning S, Wang TJ, Nilsson PM et al (2010) Plasma copeptin and the risk of diabetes mellitus. Circulation 121:2102-2108

19. Roussel R, Fezeu L, Bouby N et al (2011) Low water intake and risk for new-onset hyperglycemia. Diabetes Care 34:2551-2554

20. Bardoux P, Bichet DG, Martin H et al (2003) Vasopressin increases urinary albumin excretion in rats and humans: involvement of V2 receptors and the renin-angiotensin system. Nephrol Dial Transplant 18:497-506

21. Bankir L, Bouby N, Ritz E (2013) Vasopressin: a novel target for the prevention and retardation of kidney disease? Nat Rev Nephrol 9: 223-239

22. Meijer E, Bakker SJ, Halbesma N, de Jong PE, Struck J, Gansevoort RT (2010) Copeptin, a surrogate marker of vasopressin, is associated with albuminuria in a large population cohort. Kidney Int 77:29-36

23. Velho G, Bouby N, Hadjadj S et al (2013) Plasma copeptin and renal outcomes in patients with type 2 diabetes and albuminuria. Diabetes Care 36:3639-3645 
24. Bouby N, Bachmann S, Bichet D, Bankir L (1990) Effect of water intake on the progression of chronic renal failure in the $5 / 6$ nephrectomized rat. Am J Physiol 258:F973-F979

25. Bichet DG, Arthus MF, Barjon JN, Lonergan M, Kortas C (1987) Human platelet fraction arginine-vasopressin. Potential physiological role. J Clin Invest 79:881-887

26. Livak KJ, Schmittgen TD (2001) Analysis of relative gene expression data using real-time quantitative PCR and the 2(-delta delta $\mathrm{C}(\mathrm{T})$ ) method. Methods 25:402-408

27. Bergmeyer HU (ed) (1974) Methods of enzymatic analysis. Academic, New York

28. Perucca J, Bichet DG, Bardoux P, Bouby N, Bankir L (2008) Sodium excretion in response to vasopressin and selective vasopressin receptor antagonists. J Am Soc Nephrol 19:1721-1731

29. Morel A, O'Carroll AM, Brownstein MJ, Lolait SJ (1992) Molecular cloning and expression of a rat V1a arginine vasopressin receptor. Nature 356:523-526

30. Zerbe RL, Miller JZ, Robertson GL (1991) The reproducibility and heritability of individual differences in osmoregulatory function in normal human subjects. J Lab Clin Med 117:51-59

31. Hems DA, Whitton PD (1973) Stimulation by vasopressin of glycogen breakdown and gluconeogenesis in the perfused rat liver. Biochem J 136:705-709

32. Wideman CH, Murphy HM (1993) Modulatory effects of vasopressin on glucose and protein metabolism during food-restriction stress. Peptides 14:259-261

33. Enhorning S, Leosdottir M, Wallstrom P et al (2009) Relation between human vasopressin 1a gene variance, fat intake, and diabetes. Am J Clin Nutr 89:400-406

34. Lang F, Busch GL, Ritter M et al (1998) Functional significance of cell volume regulatory mechanisms. Physiol Rev 78:247-306

35. Cryer PE (2012) Minireview: glucagon in the pathogenesis of hypoglycemia and hyperglycemia in diabetes. Endocrinology 153:1039-1048

36. Guillon G, Grazzini E, Andrez M et al (1998) Vasopressin: a potent autocrine/paracrine regulator of mammal adrenal functions. Endocr Res 24:703-710

37. Morgan NG, Shipp CC, Exton JH (1983) Studies on the mechanism of inhibition of hepatic cAMP accumulation by vasopressin. FEBS Lett 163:277-281

38. Chabardes D, Imbert-Teboul M, Elalouf JM (1999) Functional properties of $\mathrm{Ca} 2+$-inhibitable type 5 and type 6 adenylyl cyclases and role of $\mathrm{Ca} 2+$ increase in the inhibition of intracellular cAMP content. Cell Signal 11:651-663

39. Itoh S, Yamada S, Mori T et al (2006) Attenuated stress-induced catecholamine release in mice lacking the vasopressin $\mathrm{V} 1 \mathrm{~b}$ receptor. Am J Physiol Endocrinol Metab 291:E147-E151
40. Koschorreck M, Gilles ED (2008) Mathematical modeling and analy sis of insulin clearance in vivo. BMC Syst Biol 2:43

41. Bouby N, Ahloulay M, Nsegbe E, Dechaux M, Schmitt F, Bankir L (1996) Vasopressin increases glomerular filtration rate in conscious rats through its antidiuretic action. J Am Soc Nephrol 7: 842-851

42. Bardoux P, Martin H, Ahloulay M et al (1999) Vasopressin contributes to hyperfiltration, albuminuria, and renal hypertrophy in diabetes mellitus: study in vasopressin-deficient Brattleboro rats. Proc Natl Acad Sci U S A 96:10397-10402

43. den Boer M, Voshol PJ, Kuipers F, Havekes LM, Romijn JA (2004) Hepatic steatosis: a mediator of the metabolic syndrome. Lessons from animal models. Arterioscler Thromb Vasc Biol 24:644-649

44. Wendel AA, Li LO, Li Y, Cline GW, Shulman GI, Coleman RA (2010) Glycerol-3-phosphate acyltransferase 1 deficiency in ob/ob mice diminishes hepatic steatosis but does not protect against insulin resistance or obesity. Diabetes 59:1321-1329

45. Moon YA, Liang G, Xie X et al (2012) The Scap/SREBP pathway is essential for developing diabetic fatty liver and carbohydrate-induced hypertriglyceridemia in animals. Cell Metab 15:240-246

46. Kirk CJ, Hems DA (1979) The control by vasopressin of carbohydrate and lipid metabolism in the perfused rat liver. Biochim Biophys Acta 583:474-482

47. Ma GY, Hems DA (1975) Inhibition of fatty acid synthesis and stimulation of glycogen breakdown by vasopressin in the perfused mouse liver. Biochem J 152:389-392

48. Assimacopoulos-Jeannet F, Denton RM, Jeanrenaud B (1981) Stimulation of hepatic lipogenesis and acetyl-coenzyme A carboxylase by vasopressin. Biochem J 198:485-490

49. Azizi M, Iturrioz X, Blanchard A et al (2008) Reciprocal regulation of plasma apelin and vasopressin by osmotic stimuli. J Am Soc Nephrol 19:1015-1024

50. Hus-Citharel A, Bouby N, Frugiere A, Bodineau L, Gasc JM, Llorens-Cortes C (2008) Effect of apelin on glomerular hemodynamic function in the rat kidney. Kidney Int 74:486-494

51. Galanth C, Hus-Citharel A, Li B, Llorens-Cortes C (2012) Apelin in the control of body fluid homeostasis and cardiovascular functions. Curr Pharm Des 18:789-798

52. Knauf C, Drougard A, Fournel A, Duparc T, Valet P (2013) Hypothalamic actions of apelin on energy metabolism: new insight on glucose homeostasis and metabolic disorders. Horm Metab Res 45:928-934

53. Principe A, Melgar-Lesmes P, Fernandez-Varo G et al (2008) The hepatic apelin system: a new therapeutic target for liver disease. Hepatology 48:1193-1201 